\title{
Fair and Fit Resource Allocation Algorithm for Network-Controlled Device-to-Device (D2D) Communication
}

\author{
Yusmardiah Yusuf, Darmawaty Mohd. Ali, and Roslina Mohamad
}

\begin{abstract}
Device-to-device (D2D) communication is a promising new feature for next generation cellular networks. However, enabling D2D communications in a cellular network presents a challenge in resource allocation due to the spectrum sharing with the cellular users which cause interference. The previous works in D2D resource allocation focused on improving the throughput of the overall network while completely ignoring the fairness aspect. Therefore, such issues can be managed if radio resource is assigned intelligently with the coordination from the eNodeB (eNB). In this paper, a Fair and Fit Resource Allocation (FFRA) algorithm is proposed which exploits the path loss experienced by the potential D2D user equipments (UEs) when choosing the communication path. Then, D2D UEs were scheduled using proportional fair (PF) packet scheduler. PF scheduler was developed to improved on the throughput and guarantees fairness among the flows. The FFRA scheme was applied in two communication scenarios, namely in a single D2D UE and multiple D2D UEs. OMNET++ discrete event simulator together with INET and SimuLTE framework are the platforms used to conduct the simulation. Simulation results show that, the FFRA algorithm outperform Max C/I and RR with $32.73 \%$ and $38.67 \%$ increased in total throughput while achieving excellent performance of fairness as the number of D2D UEs increases in the networks. In addition, the FFRA algorithm contributes to the lowest delay and packet loss amongst the algorithms.
\end{abstract}

Index Terms - mode selection; D2D; 5G; resource allocation algorithm; OMNET++.

\section{INTRODUCTION}

$\mathrm{D}$ EVICE-TO-DEVICE (D2D) communications in infrastructure networks have been studied actively since the 1990s [1], due to the potential to reduce delay, increase throughput, and to improve power or energy efficiency [2]. Currently, D2D communications is actively focusing on the application areas that include vehicle-to-everything (V2X) communications, wearables, autonomous ships and healthcare [3-6]. Third generation partnership project (3GPP) specified the basic functionalities for D2D communications in release 12,

This manuscript is submitted on $15^{\text {th }}$ January 2021 and accepted on 15th March 2021. This work was supported by the Ministry of Higher Education under FRGS Grant, Ministry of Higher Education Malaysia 600-IRMI/FRGS $5 / 3(047 / 2019)$. We would like to extend our acknowledgment to Universiti Teknologi Mara (UiTM) and those who have directly or indirectly contribute to this research (ymardiahyusuf@gmail.com).

1985-5389/C 2021 The Authors. Published by UiTM Press. This is an open access article under the CC BY-NC-ND license (http://creativecommons.org/ licenses/by-nc-nd/4.0/) where the main motivation was to develop a global standard for public safety communications [7]. However, the application scenario of 3GPP proximity services (ProSe) was not limited to public safety, D2D extension of conventional cellular services was also considere, hence, the new D2D based on Long Term Evolution-Advanced (LTE-A) technology was introduced in [8].

Network-controlled D2D communication is gaining rapid acceptance among researchers as a means to achieve very low latencies. In a traditional cellular network, a communication occurs between a user equipment (UE) and eNode B (eNB). Whereas in D2D communication, when two (or more) proximity UEs have discovered each other, these UEs can start communicating directly between them and reduce the delay. The physical interface between two proximity UEs is called sidelink (SL). Time-frequency resources for the SL are shared with the uplink (UL), and the SL waveform is also similar to the single-carrier frequency-division multiple access (SCFDMA) UL waveform [2]. Enabling network-controlled D2D requires solving several problems such as to discover which pairs are eligible for D2D communication due to certain rules and resource allocation management. Therefore, the resource management issue can be divided into two aspects which are mode selection and scheduling at the MAC layer [9]. Furthermore, mode selection criteria for D2D communication are not yet defined in 3GPP standards [10].

In this paper, resource allocation management was explored for network-controlled D2D communication. Thus, a Fair and Fit Resource Allocation (FFRA) algorithm was proposed which exploits the path loss experienced by the potential D2D pairs when choosing the communication path and the D2D UE is scheduled using proportional fairness $(\mathrm{PF})$ packet scheduler. PF scheduler was deployed with the aim to improve system throughput and fairness while reducing packet loss and delay among the flows. OMNET++ discrete event simulator together with INET and SimuLTE framework are the platforms to conduct the simulation for mode switching and resource allocation algorithm.

The rest of this paper is organized as follows. Section II explains on previous works on resource allocation for D2D networks. The basic principles on channel model is presented in Section III. Section IV describes the FFRA algorithm; Section $\mathrm{V}$ discusses the simulation environment and parameters used while Section VI explains on the findings. Finally, Section 
VII concludes the paper.

\section{PREVIOUS Works ON RESOURCE AllocAtion ALGORITHM}

Both academia and industry have studied transmission called mode selection (MS) for D2D communications underlaying a cellular network $[11,12]$. In these works, the transmission mode refers to the D2D mode where two potential D2D user equipments (UEs) communicate directly. As in traditional cellular networks, where two UEs communicate via the base station (BS). Mode selection procedure performs an important function in D2D transmission with the aim of improving spectral efficiency of the cellular networks [13].

Aymen Omri et al. [14] proposed a mode selection procedure for D2D communication underlaying cellular network based on predefined distance limitation between D2D users. If the actual distance is greater than the predefined value, the cellular mode is used otherwise the D2D mode is used. Researchers in [15] investigated the $\mathrm{D} 2 \mathrm{D}$ mode selection with the purpose to increase the system capacity of D2D users. The researchers used an evolutionary game-based method using the utility function that considers overall data rate of D2D users and spectrum resource utilization. By adopting the idea from [14], the mode selection was implemented by comparing received signal strength (RSS) of the three different modes such as direct reuse mode, cellular mode, and relay mode. Among these three modes, user with the highest RSS was selected to use the D2D transmission mode.

In resource allocation algorithm, after the process of mode selection, scheduling algorithm will take its responsibility to allocate the resource blocks (RBs) of the uplink (UL) subframe on each time transmission interval (TTI). Fairness is one of the performance metrics that play a vital role when managing resources in fifth generation (5G) networks [16]. Therefore, research in [17-20] adopted the proportional fair (PF) based algorithm for resource allocation in D2D transmission. For instance, an optimal proportionally fair (PF) algorithm that exploited the logarithmic sum of the user throughput was investigated in [17]. The authors proposed a heuristic algorithm based on PF scheme for downlink cellular communication, which produced a lower computational complexity algorithm. To achieve this goal, the scheduling problem was decomposed into two; one carrier-to-cellular user problem that adopted Hungarian algorithm and one carrier-to-D2D user problem. This is due to the higher priority assigned to the traditional cellular users as compared to the D2D users [5]. The researchers claimed that, heuristic algorithm was simpler than the optimal PF scheme. However, the optimal PF scheme outperformed in terms of total system throughput.

W. Zheng et al. proposed a PF-based scheduling algorithm in the orthogonal frequency-division multiple access (OFDMA) network to enhance the data rate and fairness of cellular and D2D users [18]. The researchers adopted PF algorithm in order to serve users with bad channel conditions, to allocate the resources as well as to improve the quality of service (QoS). In order to provision QoS, signal to interference noise ratio (SINR) threshold of $0 \mathrm{~dB}$ was used. Otherwise, the user transmission was considered failed. The allowable region was decided based on the transmit power limitation and QoS requirement. Then, the researchers calculated the joint transmit power for the cellular user and D2D pair in the permissible region and compared $\mathrm{PF}$ scheme with Maximum Carrier to Interference (Max C/I) scheme. The outcome revealed that more than $8 \%$ of D2D pairs and cellular users were not served when using Max C/I scheme. On the other hand, PF scheme served more users and achieved the fairness.

The investigation on scheduling algorithm based on PF scheme was extended by $\mathrm{X}$. Li et al. in [19]. The authors suggested a combination of power control and resource scheduling technique in underlay D2D transmission. The main goal was to increase the total users' proportional fairness functions and at the same time taking into consideration the fairness, interference, co-channel interference and SINR. The base station knew which D2D user should be permitted to reuse the cellular resources by referring to the SINR requirement. The D2D user was required to meet the minimum SINR requirements in order to reuse the cellular spectrum with restricted transmit power. Moreover, the QoS constraint was guaranteed through an appropriate power control technique. The presented resource allocation scheme had enhanced the data rate and improved fairness and guaranteeing the QoS performance of all D2D and cellular users.

A joint mode selection, resource allocation and scheduling algorithm was proposed in [20]. The mode selection method is according to the received signal to interference noise ratio (SINR). The mode selection was modeled using Markov chain and results were analyzed in terms of probability density function (PDF) and the cumulative distribution function (CDF) of the output SINR for the single and multi-user scenario. A proportional fair (PF) scheduler was adopted to allocate resources among all users. From the simulation, the D2D users' throughput increased as the D2D pairs increased. The proposed algorithm was compared to the optimal scheduler where the scheme achieved better spectral efficiency performance and the difference only less than $5 \%$ of the optimal scheduler..

$\mathrm{PF}$ is a channel aware scheduler. From the previous research reviews, it can be concluded that PF algorithm offers good tradeoff between throughput and fairness [20] as compared to other algorithms such as Max C/I and Round Robin (RR). Hence, PF algorithm is deployed to schedule the D2D UEs in the proposed resource allocation scheme and compare the performance with Max C/I and RR. Max C/I is also a channel aware scheduler that allocates UEs with the highest value of CQI while RR is a channel unaware scheduling scheme that serves the UEs with an equal number of resource blocks. Furthermore, RR gives good performance in terms of fairness.

\section{PRINCIPLES OF PATH LOSS MODEL}

In this section the main principles that are adopted in this paper, which is path loss model is described. Channel model is the connection between transmission and receiving power for wireless transmission presented in a mathematical formula [21]. Realistic channel model is used and urban macrocell has been deployed as the pathloss scenario in SimuLTE framework and 
it is defined and calculated based on the 3GPP standard [22]. A path loss model relates the path loss to the distance between the transmitter and the receiver where the received power, $\mathrm{P}_{\mathrm{rx}}$ is computed using (1).

$$
P_{r x}=P_{t x}-P \text { loss }-F-S
$$

Where $\mathrm{P}_{\mathrm{tx}}$ is the transmission power, Ploss is the path loss due to the distance between eNB-UE or UE-UE (which depends on frequency), $\mathrm{F}$ and $\mathrm{S}$ are the attenuation due to fast and slow fading respectively [23].

Fading in a telecommunication channel is the variation of the transmitted signal strength during propagation over time and over frequency and it occurs due to numerous reasons involving distance attenuation, obstacles, motion of transmitter and/or receiver and the arrival of multiple signals. Slow fading occurs typically due to shadowing effect when the signal is obstructed by large objects while fast fading occurs due to multipath propagation and motion of the mobile a distance comparable to the carrier wavelength [21].

\section{FFRA ALGORITHM}

A single carrier frequency division multiple access (SCFDMA) frequency-division duplexing (FDD) system is selected for D2D integration. Initially, there are two types of UEs, cellular UE and potential D2D UE. The cellular UE uses the uplink (UL) for data transmission while UE in D2D mode employed the sidelink (SL). However, the UEs in D2D mode are also connected to eNB as eNB is still in charge of data plane control for UL subframe. In D2D transmission, transmitting UE can send a packet to receiving UE without using traditional path. ENB can grant transmitting UE with resources for data transmission and command potential receiving UE to listen on the same RB for reception.

The main parameter used for the mode selection (MS) algorithm was path attenuation or path loss. In this paper, MS scheme exploits path loss which was experienced by the UEs. In the beginning of the simulation, a pair of UE which consist of D2D transmitter and D2D receiver was used as a potential D2D UE when the D2D transmitter had buffered data for a targeted D2D receiver in proximity. MS algorithm was applied in the initial stage where the decision was made by eNB whether the potential D2D UE will transmit the data using SL (D2D transmission) or using UL (cellular transmission). Therefore, the selection of transmission mode was based on the comparison of path loss experienced between SL and UL channel. At first, a pair of potential D2D UE makes a decision for a mode change attempt to SL mode when;

$$
\left(\mathrm{P}_{\text {lossSL }}<\mathrm{P}_{\text {lossUL }}\right)
$$

If the pathloss in SL channel ( $\mathrm{P}_{\text {lossSL }}$ ) for potential D2D pairs is lower than the pathloss of UEs to the eNB ( $\left.\mathrm{P}_{\text {lossUL }}\right)$, then the potential D2D pairs will choose SL as the transmission path. The scenario for the MS scheme is illustrated in Fig. 1.

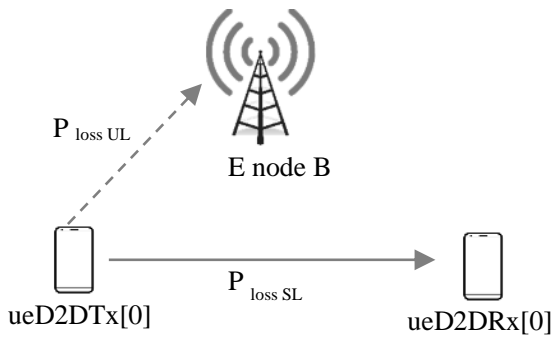

Fig. 1.Mode selection scenario

In the next stage, after mode of transmission was selected, all the D2D UEs were sorted in a single list by using packet scheduler. D2D UEs were arranged based on metrics value which was calculated using (2). The packet scheduler was responsible for resource allocation of uplink subframe on each TTI to all the UEs in the same cell. In this paper, the conventional proportional fair (PF) scheduler was deployed to serve the UE. This algorithm allocates UE based on the ratio of channel conditions which is calculated using metrics in (3). The $\mathrm{PF}$ algorithm is aimed to provide a balance between throughput and fairness performance in the networks and at the same time fulfilled the QoS requirements.

$$
M_{i, k}^{P F}=\frac{r i(t)}{R i(t)}
$$

$r i(t)$ means the achievable data rate according to the instantaneous channel quality of user $i$ at t-th TTI, $R_{i}(t)$ is the past average data rate achieved by $i_{\text {th }}$ user until at time $t_{\text {th }}$ and $\beta$ is a variable ranging from 0 to $1 . R_{i}(t)$ is calculated using (4).

$$
R_{i}(t)=\beta R_{i}(t-1)+(1-\beta) r_{i}(t)
$$

At each subframe, the average rate is updated according to (4) where $\beta$ is ranging from $0<\beta<1$. Thus, this method maintains fairness while trying to schedule the user with the best channel condition.

In summary, the MS is performed using the decision rule of (2) and the eNB periodically runs an MS algorithm that selects the communication mode for the potential D2D UEs. The next step was resource allocation and scheduling scheme. For each subchannel, the PF metrics for all D2D UEs was calculated using (3) and sorted the D2D UEs in decreasing order. The D2D UE with the highest PF metric was selected and all the UEs were scheduled until no more available resource blocks (RBs). In the case of cellular transmission, the UE that experienced higher path loss $\left(\mathrm{P}_{\text {lossUL }}>\mathrm{P}_{\text {lossSL }}\right)$ will not be scheduled in the same subframe, since frequency reuse is not considered in the simulation. The summary of the FFRA algorithm and flow chart of the simulation is explained in Algorithm 1 and Fig. 2 respectively. 


\begin{tabular}{l}
\hline Algorithm 1: FFRA algorithm \\
\hline Stage 1: Mode selection \\
For all potential D2D pairs; \\
Calculate path loss according to realistic channel model \\
according to rule (1) \\
For all potential D2D UEs, the D2D mode is determined \\
using (2). \\
Stage 2: Scheduling algorithm \\
For available number of subchannel; \\
Calculate PF metrics for all UEs using (3); \\
Sort flows by decreasing PF metrics \\
While flow list is not empty, extract UEs from top of the list \\
and served the UEs based on the requested RBs until no more \\
RBs are available
\end{tabular}

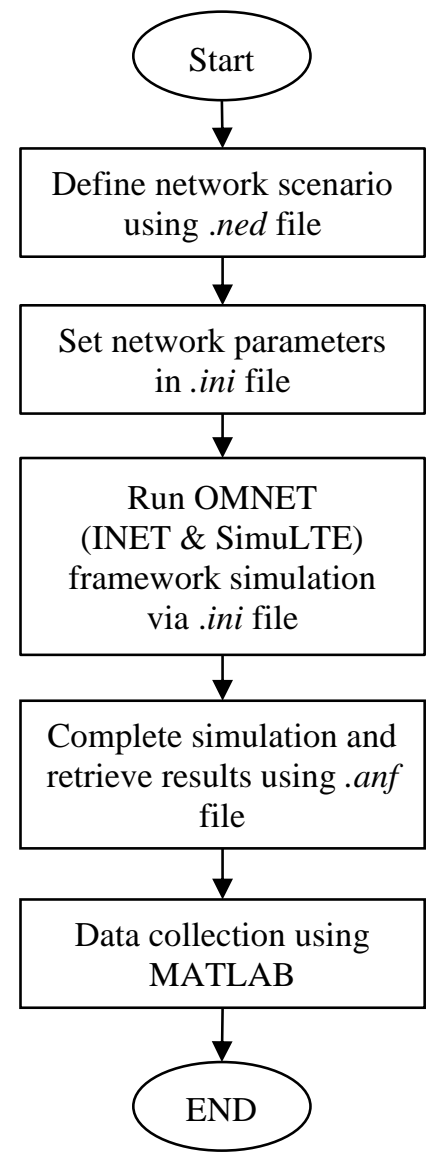

Fig. 2.Flow chart of the simulation

\section{SimUlation ENVIRONMENT AND PARAMETERS}

A single cell that consists of one eNB was considered in the simulation. Initially, UEs were divided into two groups named $u e D 2 D T x$ and $u e D 2 D R x$ that represent transmitting D2D UEs, and receiving D2D UEs. The simulation environment in [24] was refered by utilizing different parameters such as mode selection approach, scheduling scheme and mobility model. The FFRA algorithm was compared with the algorithm in [24] and round robin (RR). The algorithm in [24] used the maximum carrier to interference ratio (Max C/I) algorithm to allocate RBs for D2D UEs while guaranteeing the channel quality indicator
(CQI) as the requirements for potential D2D UEs. However, Max C/I algorithm ignores the system fairness. In this paper, the simulation consists of two different environments which are the mode switching and resource allocation algorithm.

\section{A. Effect of mode switching}

Firstly, in order to demonstrate the result of mode switching process for MS algorithm, the network environment shows in Fig. 3 was considered and a single pair of D2D UE which consists of one transmiting and receiving UE was simulated respectively. Transmitting and receiving UE was sending one packet every $10 \mathrm{~ms}$ of a VoIP traffic. The packet length were varied from 200 bytes to 1200 bytes to assess the performance of the D2D flow with different traffic loads. Initially, transmitting UE is $100 \mathrm{~m}$ away from eNB and $20 \mathrm{~m}$ from the receiving UE. The UEs swing back and forth in a linear motion at a speed of $3 \mathrm{~m} / \mathrm{s}$. This path allows direct link between the UEs so that the UEs would experienced different channel quality during the simulation. In this analysis, the scenario where MS was enabled is compared with the flow that used the infrastructure link where MS was disabled.

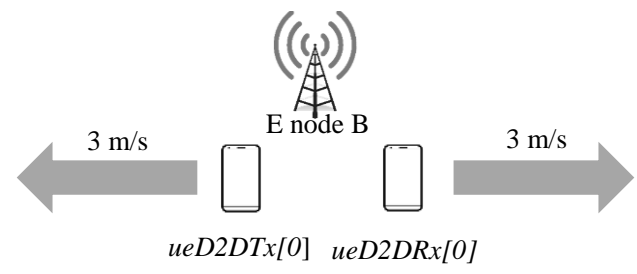

Fig. 3.Simulation environment

\section{B. Mode selection algorithm comparison}

Secondly, the number of D2D pairs were varied from 10 to 50 pairs and positioned similarly as in Fig. 2. The aim is to observed the effect of the proposed resource allocation algorithm when a large number of UEs are active in the same cell. The proposed algorithm was compared with Max C/I [24] and RR. The UEs were established with VoIP application that consist of VoIP sender and VoIP receiver. In this case, 500 bytes size of talk packets were fixed with $10 \mathrm{~ms}$ send interval. The simulation parameter is summarized in table 1 .

TABLE 1

SIMULATION PARAMETER

\begin{tabular}{ll}
\hline \hline Parameter & Value \\
\hline Bandwidth & $10 \mathrm{MHz}$ \\
Number of RBs & 50 \\
eNB Transmit power & $26 \mathrm{~dB}$ \\
Mobility model & Linear (INET) \\
Traffic flows & VoIP \\
Number of D2D pairs & $10-50$ \\
Simulation time & $30 \mathrm{~s}$ \\
Number of independent replicas & 10 \\
\hline \hline
\end{tabular}

\section{RESULT AND DISCUSSION}

In this section the performance of the proposed MS is analysed when the mode switching is deployed and compared with Max C/I approach [24]. This section is divided into two subsections. The first subsection describes the proposed MS 
algorithm when MS is enabled and disabled to record the effect of mode switching mechanism when the number of packet size are varied. The analysis has been evaluated in terms of throughput, CQI and delay performance.

Secondly in subsection $B$ explains the FFRA algorithm when it is compared with other algorithms. The comparison has been made in terms of throughput, fairness, delay, and packet loss. Both simulations employed VoIP application using user datagram protocol (UDP) as the transport protocol. The results are compared with 3GPP QoS requirement for mobile networks.

\section{A. Mode Switching Analysis}

Fig. 4 illustrates the graph for CQI experienced by the transmitting UE. Its is noticed that, when the mode selection is enabled, the CQI gives an excellent channel quality since the flow periodically switches from the sidelink to the uplink and vice versa. As a result, it is observed that by enabling the mode selection, the scheme allows the flow to use lowest path loss, better CQI, hence using better modulation for transmission.

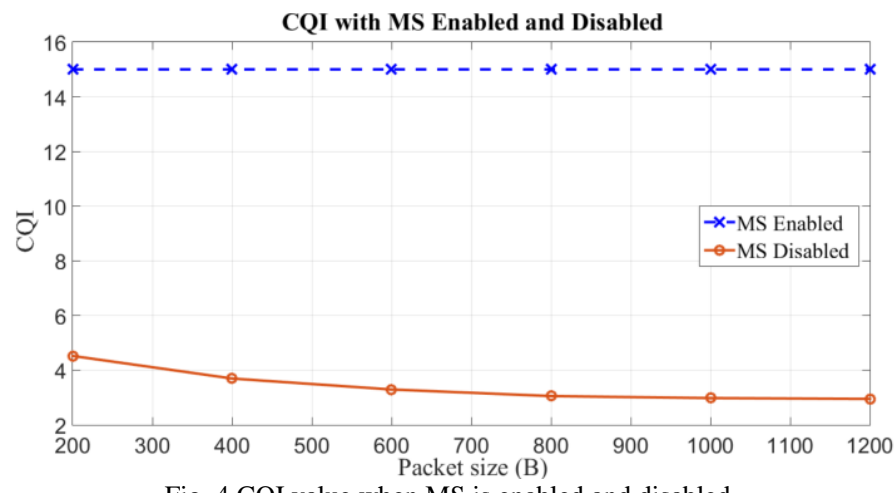

Fig. 4.CQI value when MS is enabled and disabled

In terms of delay parameter with different packet lengths as shown in Fig. 5, the delay is increased drastically at the points where the CQI is small that is when mode selection is not active. This behavior is more noticeable when the traffic load increases which is at 800 bytes due to larger packets being transmitted. On the other hand, when mode selection is considered allows the flow to maintain the latency. The QoS value for the VoIP delay is set to be less than $100 \mathrm{~ms}$ respectively as specified by the 3GPP standard [25]. Therefore, the MS deployed in the algorithm contributed to lower delay, and is in the acceptable range to provision the Quality of User Experience (QoE).

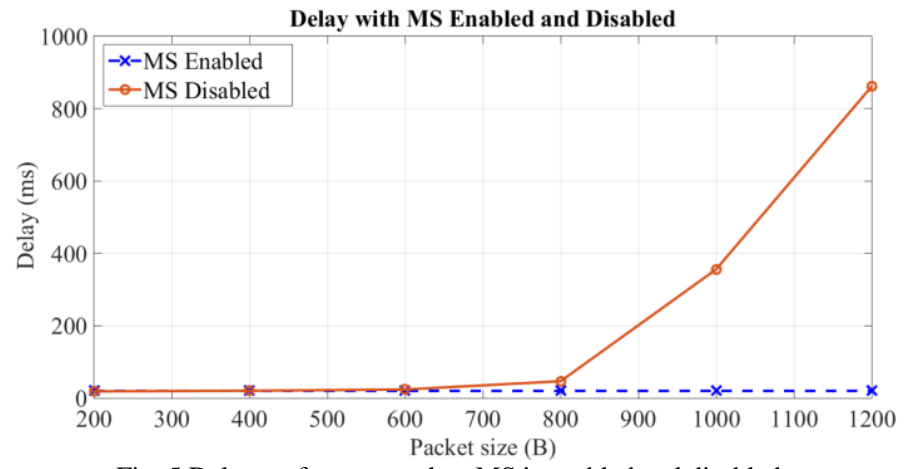

Fig. 5.Delay performance when MS is enabled and disabled
Fig. 6 illustrates the effect of MS algorithm when the distance between transmitting and receiving UE are increased from 10 $\mathrm{m}$ to $100 \mathrm{~m}$. It can be seen that when D2D distance is below 30 $\mathrm{m}$, D2D has good channel quality and the throughput is maintained. However, when the D2D distance is $40 \mathrm{~m}$ to 100 $\mathrm{m}$, the D2D link started to drop meaning that low modulation technique is being used, resulting throughput degradation for D2D UEs. It can be deduced that, with the increase of D2D distance, poor channel quality might occur, resulting in higher path loss and the potential D2D pair is expected to be scheduled in uplink transmission thus throughput for D2D link degrades.

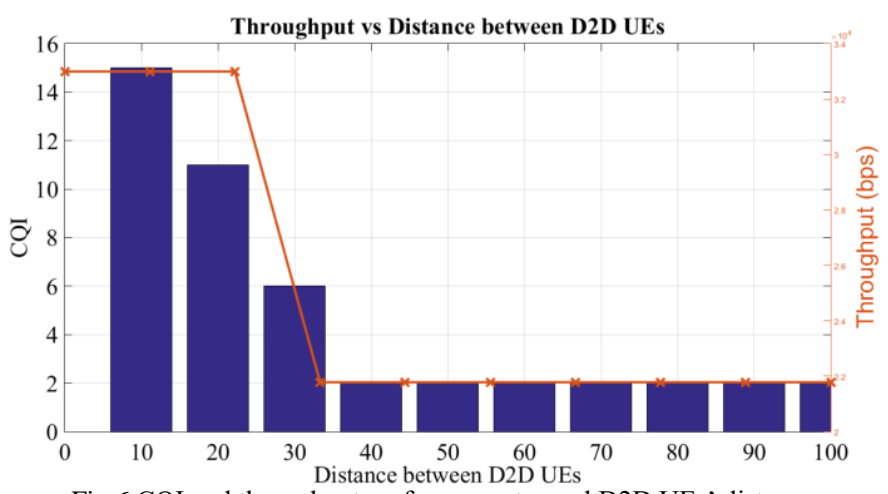

Fig 6.CQI and throughput performance toward D2D UEs' distance

\section{B. Mode Selection Performance Comparison}

In this paper, FFRA algorithm is aimed to increased the total throughput, improve delay and packet loss in the network. The throughput presents the rate of the successful packet being delivered over the physical channel. Therefore, the simulation is extended to compare the FFRA algorithm with Max C/I [24] and RR. Fig. 7 demonstrates the throughput performance when the number of D2D UEs is increased. It is observed that as the number of users increases, the throughput of VoIP for all algorithms gradually increases and shows similar trending. In addition, the throughput for FFRA algorithm is $32.73 \%$ and $38.67 \%$ higher than Max C/I and RR due to fair allocation of RBs among the users. This proved that FFRA algorithm can significantly increased the overall system throughput.

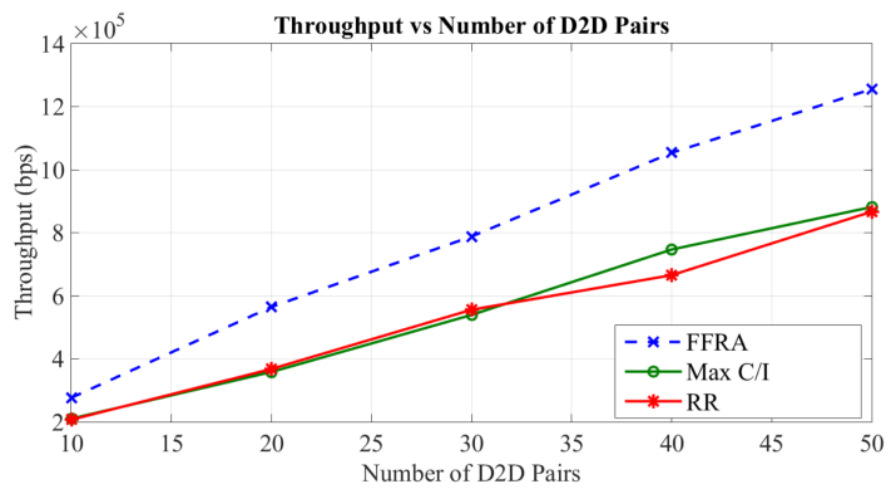

Fig. 7.Throughput performance of different algorithms

The Jain's fairness index is presented in Fig. 8. From the figure, FFRA gives the highest fairness value because the algorithm allocates resources to the user based on the ratio of the channel condition. Hence, the fairness is proportional to the channel condition. The RR algorithm assigned resources to user 
without considering the channel conditions. The fairness is reduced as the D2D pairs increased which is from 0.9 to 0.86 . However, the fairness value for Max C/I decreases at 40 to 50 D2D pairs because resources are given only to users with the best channel condition and those with low channel quality will suffer. Whereas, the fairness for FFRA is maintained between 0.92 to 0.97 because the algorithm delivers higher throughput as illustrated in Fig. 7 and thus provide a balance between throughput and fairness. It can be concluded that FFRA algorithm is fairer than Max C/I and RR.

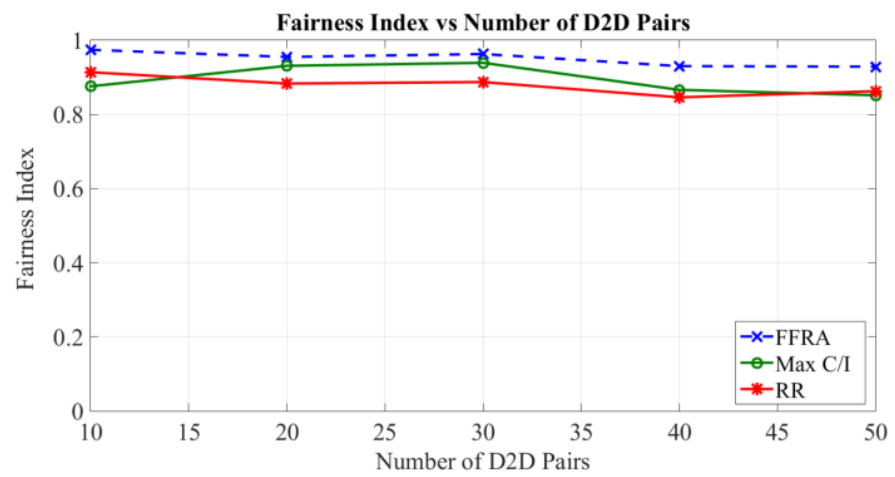

Fig. 8. Fairness performance of different algorithms

Real time service such as VoIP is an application that has strict delay requirement, which needs to be provisioned. Fig. 9 shows the delay analysis for the application used in this simulation. It is noticed that the D2D UEs suffer longer packet arrival rate in accordance to the increase in the number of users for Max C/I and RR algorithm while FFRA algorithm delivers the lowest delay which is approaching zero. It is showed that the delay for FFRA algorithm decreases by $17.76 \%$ and $24.28 \%$ than Max C/I and RR. The voice packet of all the algorithms can be transmitted because the delay is within the acceptable range as specified by the $3 \mathrm{GPP}$ for mobile communication which is $100 \mathrm{~ms}$.

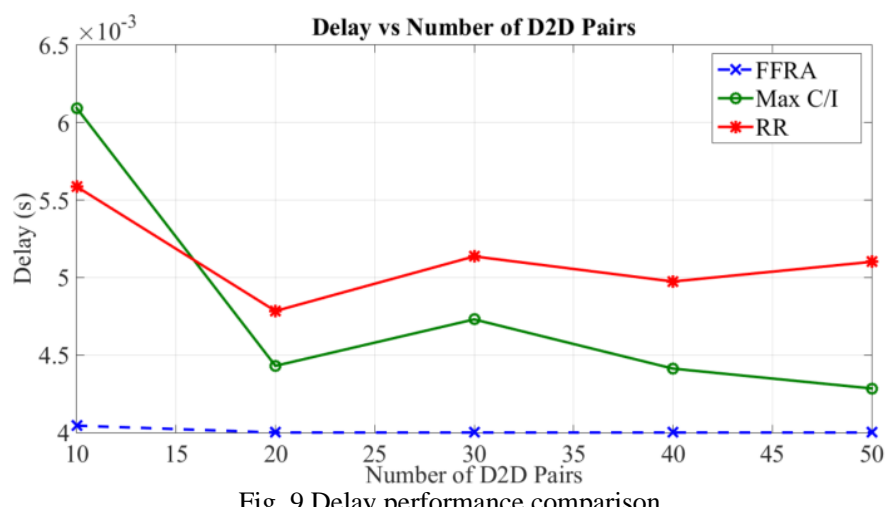

Fig. 9.Delay performance comparison

In SimuLTE, the packet loss is measured according to the hybrid automatic repeat request (HARQ) eror which means error to decoding. This HARQ error gives the percentage of errors due to HARQ over the total transport block received. Therefore, packet loss is an important parameter to verify whether the packets are delivered on time. Fig. 10 shows the packet loss for all the algorithms. It is noticed that the packet loss for FFRA algorithm is $97.12 \%$ and $96.54 \%$ lower than Max $\mathrm{C} / \mathrm{I}$ and RR and approaching zero when D2D UEs increases.
The acceptable range of packet loss for VoIP as specified by 3GPP should not exceed $1 \times 10^{-2}$ [25]. However, it is observed that the Max C/I and RR algorithm has violated the QoS value specified by $3 \mathrm{GPP}$ standard. The Max C/I and RR schemes have higher packet loss and this is in accordance with the throughput performance as in Fig. 7. Throughput decreases due to some packet is not received in a given time. As a result, the possibility to discard packets for deadline expiration increases.

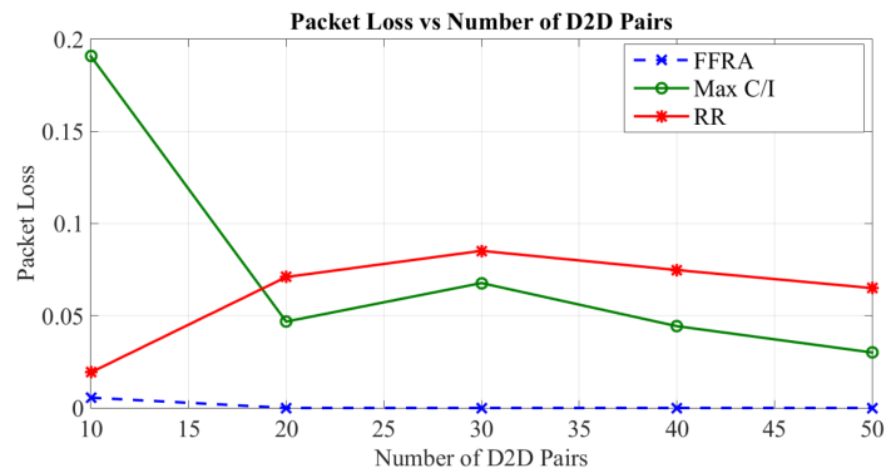

Fig. 10.Packet loss performance of different algorithms

\section{CONCLUSION}

In this paper, FFRA for network-controlled D2D communication is proposed. The technique selects the mode of transmission that offers an acceptable performance according to the path loss experienced by the potential D2D UEs. Whereas the scheduling for D2D UEs is performed using PF algorithm that offers a balance between throughput and fairness performance. From the analysis, FFRA algorithm outperformed the Max C/I and RR algorithm in terms of throughput, fairness, delay and packet loss. The throughput for the proposed algorithm improved $32.73 \%$ and $38.67 \%$ than Max C/I and RR while achieving excellent performance of fairness as the number of D2D UEs increases. In terms of delay and packet loss, FFRA delivers the lowest when increasing number of D2D UEs are distributed in the cell.

For future recommendation, FFRA algorithm could be enhanced by exploiting frequency reuse between D2D UEs in order to improve the spectral efficiency. In addition, when reuse is allowed, interference will be the main issue and it is crucial to be managed.

\section{ACKNOWLEDGMENT}

This work was supported by the Ministry of Higher Education under FRGS Grant, Ministry of Higher Education Malaysia 600-IRMI/FRGS 5/3 (047/2019). We would like to extend our acknowledgment to Fakulti Kejuruteraan Elektrik Universiti Teknologi Mara (UiTM) Shah Alam and those who have directly or indirectly contribute to this research.

\section{REFERENCES}

T. N. Adachi, M. , "A study on channel usage in a cellular Ad-Hoc united communication system," IEICE Trans. Communication, vol. 81, pp. 1500-1507, 1998.

[2] M. Höyhtyä, O. Apilo, and M. Lasanen, "Review of Latest Advances in 3GPP Standardization: D2D Communication in 5G Systems and Its Energy Consumption Models," Future Internet, vol. 10, no. $1,2018$. 
[3] M. Höyhtyä, J. Huusko, M. Kiviranta, K. Solberg, and J. Rokka, "Connectivity for Autonomous Ships: Architecture, Use Cases, and Research Challenges," presented at the 2017 International Conference on Information and Communication Technology Convergence (ICTC), Jeju, South Korea, 2017.

[4] P.-Y. Kong, "Cellular-Assisted Device-to-Device Communications for Healthcare Monitoring Wireless Body Area Networks," IEEE Sensors Journal, vol. 20, no. 21, pp. 13139-13149, 2020.

[5] F. Yucel, A. Bhuyan, and E. Bulut, "Secure, Resilient and Stable Resource Allocation for D2D-based V2X Communication," Salt Lake City, UT, USA, USA, 2020.

[6] S. Saafi, Hosek, and A. Kolackova, "Cellular-enabled Wearables in Public Safety Networks: State of the Art and Performance Evaluation," presented at the 12th International Congress on Ultra Modern Telecommunications and Control Systems and Workshops (ICUMT), Brno, Czech Republic, 2020.

[7] 3GPP, "Proximity-based services (ProSe); Release 12)," in "3GPP TS 23.303 version 12.2.0 Release 12)," Jun. 2014 2014, vol. V12.1.0.

[8] 3GPP, "LTE Network architecture," in "3GPP TS 23.002 version 12.6.0 Release 12," 2015.

[9] G. Nardini, G. Stea, A. Virdis, D. Sabella, and M. Caretti, "Resource allocation for network-controlled device-to-device communications in LTE-Advanced," Wireless Networks, vol. 23, no. 3, pp. 787-804, 2016.

[10] U. N. Kar and D. K. Sanyal, "A Critical Review of 3GPP Standardization of Device-to-Device Communication in Cellular Networks," SN Computer Science, vol. 1, no. 1, 2019.

[11] Y. Liu, "Optimal Mode Selection in D2D-Enabled Multibase Station Systems," IEEE Communications Letters, vol. 20, no. 3, pp. 470-473, 2016.

[12] S. Maghsudi and D. Niyato, "On Transmission Mode Selection in D2D-Enhanced Small Cell Networks," IEEE Wireless Communications Letters, vol. 6, no. 5, pp. 618-621, 2017.

[13] P. Mach, Z. Becvar, and T. Vanek, "In-Band Device-to-Device Communication in OFDMA Cellular Networks: A Survey and Challenges," IEEE Communications Surveys \& Tutorials, vol. 17, no. 4, pp. 1885-1922, 2015.

[14] A. Omri and M. O. Hasna, "A Distance-Based Mode Selection Scheme for D2D-Enabled Networks With Mobility," IEEE Transactions on Wireless Communications, vol. 17, no. 7, pp. 43264340, 2018.

[15] Y. Li, W. Song, Z. Su, L. Huang, and Z. Gao, "A Distributed Mode Selection Approach Based on Evolutionary Game for Device-toDevice Communications," IEEE Access, vol. 6, pp. 60045-60058, 2018.

[16] N. DoCoMo, "5G Radio Access: Requirements, Concept and Technologies," in "White Papers," 2014.

[17] J. Gu, S. J. Bae, S. F. Hasan, and M. Y. Chung, "Heuristic Algorithm for Proportional Fair Scheduling in D2D-Cellular Systems," IEEE Transactions on Wireless Communications, vol. 15, no. 1, pp. 769780, 2016.

[18] W. Zheng, J. Hu, C. Liu, and Y. Fu, "Cellular-D2D resource reuse algorithms based on proportional fairness," EURASIP Journal on Wireless Communications and Networking, vol. 2017, no. 1, 2017.

[19] X. Li, R. Shankaran, M. A. Orgun, G. Fang, and Y. Xu, "Resource Allocation for Underlay D2D Communication With Proportional Fairness," IEEE Transactions on Vehicular Technology, vol. 67, no. 7, pp. 6244-6258, 2018.

[20] P. S. Bithas, K. Maliatsos, and F. Foukalas, "An SINR-Aware Joint Mode Selection, Scheduling, and Resource Allocation Scheme for D2D Communications," IEEE Transactions on Vehicular Technology, vol. 68, no. 5, pp. 4949-4963, 2019.

[21] Z. M. Myo and M. T. Mon*, "Analysis of Signal Variation Based on Path Loss in LTE Network," Springer International Publishing Switzerland, 2015.

[22] I.-R. M.2135, "Guidelines for evaluation of radio interface technologies for IMT-Advanced," 2008.

[23] G. Nardini, G. Stea, and A. Virdis, "SimuLTE - A Modular Systemlevel Simulator for LTE/LTE-A Networks based on OMNeT++," in Proceedings of the 4th International Conference on Simulation and Modeling Methodologies, Technologies and Applications, 2014, pp. 59-70: Science and Technology Publications, Lda.

[24] A. Virdis, G. Nardini, and G. Stea, "Cellular-Networks Simulation Using SimuLTE," in Recent Advances in Network
Simulation(EAI/Springer Innovations in Communication and Computing, 2019, pp. 183-214.

[25] G. T. 23.203, "Digital cellular telecommunications system (Phase 2+); Universal Mobile Telecommunications System (UMTS); LTE; Policy and charging control architecture " 2010.

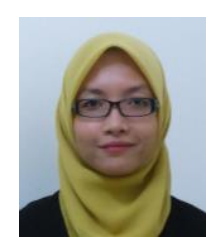

Yusmardiah Yusuf is currently a $\mathrm{PhD}$ students at Universiti Teknologi MARA. Her $\mathrm{PhD}$ research in wireless communication on Device to Device communication, resource allocation and scheduling algorithm

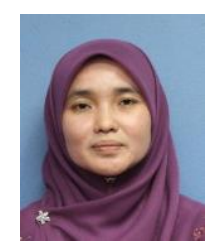

Darmawaty Mohd Ali is an Associate Professor at University Teknologi MARA (UiTM), Selangor, Malaysia. She obtained her Ph.D in 2012 from Universiti Malaya, Malaysia. She has received her Master of Engineering in Electrical in 2002 from Universiti Teknologi Malaysia. Previously, she obtained her first degree from Universiti Kebangsaan Malaysia with Honours, in Electrical, Electronic and System, graduating in 1999. She is the head of Wireless Communication Technology (WiCOT) Research Interest Group (RIG) and her research interests include Wireless.

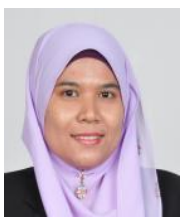

Roslina Mohamad obtained the B. Eng. degree in Electrical Engineering and M. Eng. Science degree from Universiti Malaya, Kuala Lumpur in 2003 and 2008 respectively. She received the $\mathrm{PhD}$. degree in Aerospace Engineering from Universiti Putra Malaysia, in 2016. From 2006 until now, she is with Faculty of Electrical Engineering, Universiti Teknologi MARA, as a senior lecturer. Her research interests are in computing algorithm and digital signal processing for deep space communication, channel coding, theory of computation, and wireless communication 